\title{
Data-driven discovery of the heat equation in an induction machine via sparse regression
}

\author{
Saeideh Khatiry Goharoodi*†, Pieter Nguyen Phuc* ${ }^{* \dagger}$, Luc Dupre ${ }^{\dagger}$, Guillaume Crevecoeur* ${ }^{* \dagger}$ \\ ${ }^{*}$ EEDT-DC, Flanders Make, the strategic research centre for the manufacturing industry in Flanders, Belgium \\ ${ }^{\dagger}$ Department of Electrical Energy, Metals, Mechanical Constructions and Systems (EEMMeCS) \\ Ghent University \\ Ghent, Belgium \\ Saeideh.KhatiryGoharoodi@ugent.be
}

\begin{abstract}
Discovery of natural laws through input-output data analysis has been of considerable interest during the past decade. Various approach among which the increasingly growing body of sparsity-based algorithms have been recently proposed for the purpose of free-form system identification. There has however been limited discussion on the performance of these approaches when applied on experimental datasets. The aim of this paper is to study the capability of this technique for identifying the heat equation as the natural law of heat distribution from experimental data, obtained using a Totally-Enclosed-Fan-Cooled (TEFC) induction machine, with and without active cooling. The results confirm the usefulness of the algorithm as a method to identify the underlying natural law in a physical system in the form of a Partial Differential Equation (PDE).
\end{abstract}

Index Terms-sparse regression, data-driven discovery, sparse group Lasso, heat equation

\section{NOMENCLATURE}

T Temperature.

$t \quad$ Time.

$z \quad$ Axial coordinate.

$\rho \quad$ Mass density.

$c_{p} \quad$ Specific heat capacity.

$q \quad$ Internal heat source.

$k \quad$ Thermal conductivity.

$\alpha \quad$ Thermal diffusivity.

\section{INTRODUCTION}

In spite of significant advances in automated techniques for the purpose of data generation and data acquisition, automated discovery of the underlying analytical physical laws of a system is yet a challenging area in the field of system identification. As part of a major advancement in 2009, application of symbolic regression for determining the analytical laws behind natural physical phenomena was examined [1]. The authors adopted the ability of evolutionary computation to construct the model based on a combination of several mathematical building blocks such as constants, algebraic operators, state variables and analytical functions. In this scheme, both the

This work was supported by the strategic basic research project EMODO and MoForM, and the ICON project MultiSensor of Flanders Make, the Strategic Research Centre for the Manufacturing Industry; and the FWO research project G.0D93.16N. We would like to thank Hendrik Vansompel and Dimitar Bozalakov for their assistants in capturing and processing the measurements. form and parameters of the model were a matter of search. Experimental data captured from a chaotic double pendulum was used to validate the approach. The method seems to be reliable and the identified laws are comprehensive. Nonetheless, symbolic regression is expensive and thus the method fails to scale well to big data sets.

A more recent approach by Brunton [2] proposes a sparsity based method that extracts dynamical system models in the form of ordinary differential equations (ODEs) from a predefined set of trial functions. This set is characterized with all candidate functions of the state variables that are possible to structure the model. Subsequently, sparsity-promoting techniques are applied to identify the relevant terms by solving the optimization problem. During the last two years, this approach has been improved to be used for identification of abrupt changes in a nonlinear system [3], and to have better model predictive control [4]. In addition, this technique has been further extended to encompass Partial Differential Equations (PDEs) [5] and [6]. To achieve this, time series measurements in the spatial domain are required, then a small number of spatially positioned points are used to compute the derivatives. The difficulty of numerical differentiation for noisy data and the decline of performance for highly correlated data are among the main challenges of this identification technique, although the latter challenge has been addressed recently in [7], where the optimization problem is reformulated from least absolute shrinkage and selection operator (Lasso) [8] to group Lasso [9].

The sparse regression technique has been tested on various sets of numerical data, including the chaotic Lorenz system [2]. However, there has been little discussion on the performance of this technique when applied on data obtained from experiments. In this study, we use experimental data obtained using a Totally-Enclosed-Fan-Cooled (TEFC) induction machine to evaluate the capability of this technique for identifying the heat equation as the natural law of heat distribution. Although identification of the coefficients of the heat equation using conventional parameter identification methods has been studied earlier [10] and [11], to the best of our knowledge, no analysis has been conducted to drive the form of this equation as the physical law of a system. Accordingly, using limited experimental data points, we discover the heat equation as a 
TABLE I

SPECIFICATIONS OF THE INDUCTION MACHINE

\begin{tabular}{|l|r|}
\hline Number of pole pairs $N_{p}$ & 2 \\
Nominal power $P_{N}$ & $5.5 \mathrm{~kW}$ \\
Nominal speed $n_{N}$ & $1460 \mathrm{rpm}$ \\
Nominal current $I_{N}$ & $18.6 / 10.7 \Delta / \mathrm{Y} \mathrm{A}$ \\
Nominal voltage $U_{N}$ & $230 / 400 \Delta / \mathrm{Y} \mathrm{V}$ \\
Efficiency $\eta$ & $89.6 \%$ \\
Mutual inductance $L_{m}$ & $146.7 \mathrm{mH}$ \\
Stator inductance $L_{s}$ & $153 \mathrm{mH}$ \\
Rotor inductance $L_{r}$ & $153.3 \mathrm{mH}$ \\
Stator resistance $R_{s}$ & $0.625 \Omega$ \\
Rotor resistance $R_{r}$ & $0.469 \Omega$ \\
\hline
\end{tabular}

PDE, by means of a modified version of the sparse regression technique.

\section{EXPERIMENTAL SETUP}

The experimental setup consists of a TEFC squirrel-cage induction machine with 4 poles and a $5.5 \mathrm{~kW}$ power rating, connected directly to a $50 \mathrm{~Hz} / 400 \mathrm{~V}$ main grid (see Fig. 1). The motor runs in a no-load condition and has an external cooling ventilator attached to its end, allowing to conduct the experiments both with and without active cooling of the motor. The specifications of the $5.5 \mathrm{~kW}$ induction machine are listed in Table I.

Two experiments are conducted to collect temperature data from the windings of the induction motor. For the first experiment, both the motor and the cooling ventilator operate for 3.595 hours, such that the motor is actively cooled while it runs in no-load condition. After 3.595 hours, both the motor and the ventilator are shut off and the motor is allowed to cool down without active cooling from that point onward. For the second experiment, only the motor operates, running in noload condition without active cooling. After 6.61 hours, the motor is shut off and is allowed to cool down naturally.

The temperature data is collected using 4 RTD temperature sensors, embedded into the stator windings of the induction motor. These sensors are of the type PT1000 class A, with a specified tolerance ranging between $0.15{ }^{\circ} \mathrm{C}$ and $0.55{ }^{\circ} \mathrm{C}$ for measurements between $0{ }^{\circ} \mathrm{C}$ and $200{ }^{\circ} \mathrm{C}$, as specified according to IEC 60751 [12]. Implementation of the sensors necessitates dismantling of the motor, removal of the copper windings and resin by oven heating and rewinding the stator together with the temperature sensors. In this paper, sensors at 4 locations within the stator winding have been chosen for the measurement procedure, which is shown in Fig. 2. All four sensors are located in the upper region of the motor, with two of them implemented in the stator winding itself (T2 and T3) and the other two in the end windings (T4 at the drive side, $\mathrm{T} 1$ at the fan side). The axial distance between the sensors is also depicted in Fig. 2. The radial position of all sensors is approximately at the mid-position of the stator slot. Furthermore, any difference in temperature due to the small circumferential distance between the sensors is assumed to be negligible because the setup is fully symmetric.

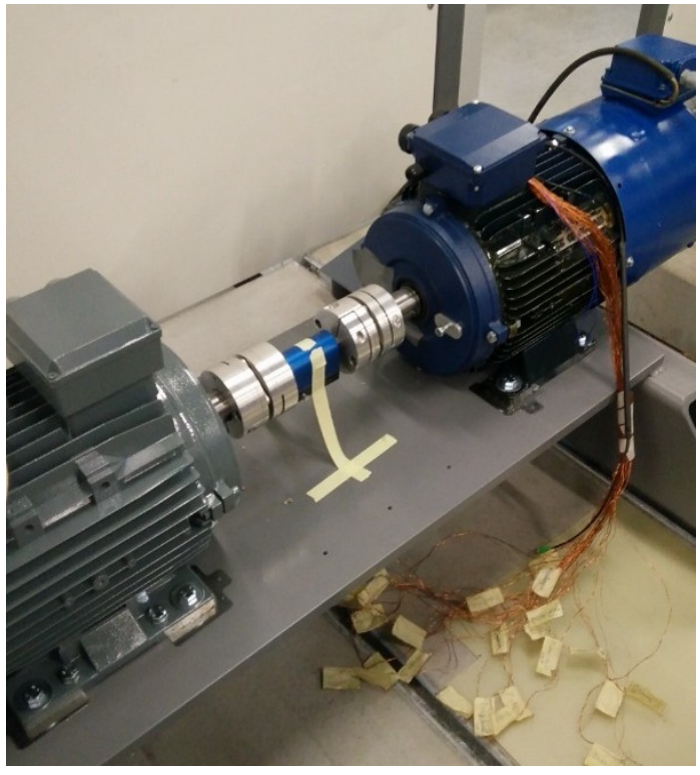

Fig. 1. Illustration of the experimental setup: the blue $5.5 \mathrm{~kW}$ test motor on the right is connected directly to a 3-phase $400 \mathrm{~V}$ main grid and runs in a noload condition, while the motor on the left is not powered. The coated copper wires coming out of the right motor are the lead wires of the temperature sensors embedded in the windings of the machine (see Fig. 2 for sensor locations).

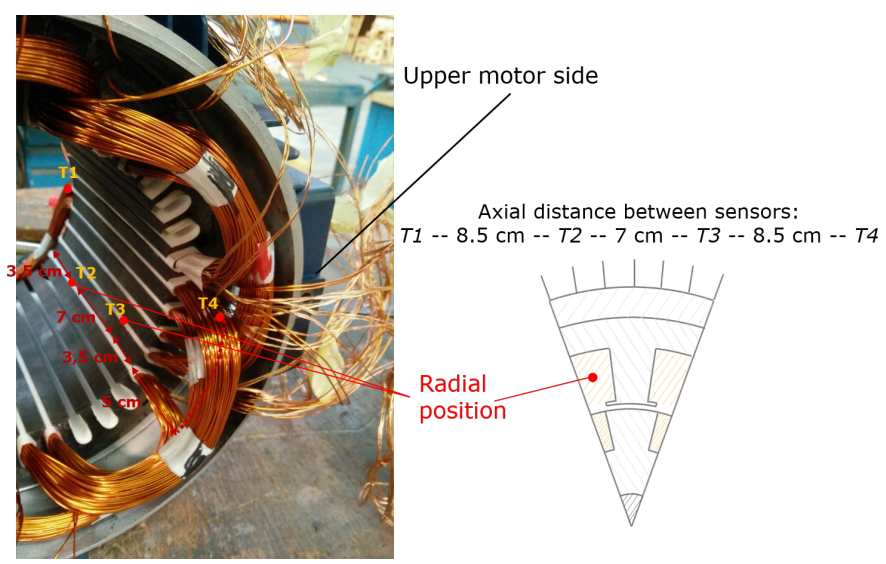

Fig. 2. Illustration of the four temperature sensor locations. Considering the symmetry of the setup, the difference in temperature due to the circumferential distance between the sensors is assumed to be negligible.

The relation between temperature and resistance of the PT1000 sensor is known from the Callendar-Van Dusen equation [12]:

$$
\begin{aligned}
R(T) & =R_{0}\left(1+A \cdot T+B \cdot T^{2}\right) \\
A & =3.9083 \cdot 10^{-3} \\
B & =-5.775 \cdot 10^{-7}
\end{aligned}
$$

where $\mathrm{T}$ denotes the temperature expressed in ${ }^{\circ} \mathrm{C}, \mathrm{R}(\mathrm{T})$ is the resistance of the RTD at temperature $\mathrm{T}$ with $\mathrm{R}_{0}=1000 \Omega$ 
being the resistance at $0^{\circ} \mathrm{C}$. The coefficients $\mathrm{A}$ and $\mathrm{B}$ in (1) were determined from the IEC 60751 norm [12]. To measure the resistance, we adopt the 4 wire measurement principle [13]: one pair of wires acts as a low-impedance current source while the other pair is used for a high-impedance measurement of the voltage over the sensor, eliminating the error due to heat-up of the lead wires.

Power supply, voltage measurement, signal amplification and linearization of the relation between measured temperature and output voltage are done in an analog fashion with an inhouse designed circuit board. The output voltage ranges from $0.5 \mathrm{~V}$ to $4.5 \mathrm{~V}$, which corresponds to a measured temperature of $0{ }^{\circ} \mathrm{C}$ and $200{ }^{\circ} \mathrm{C}$ respectively. Data acquisition is done using a MicroLabBox system from dSPACE, with a Real-Time Interface (RTI) acting as the link between the MicroLabBox hardware and the programmable software in Matlab Simulink. PC communication and data visualization are possible with software ControlDesk 6.1. A $10 \mathrm{~Hz}$ low-pass filter and $50 \mathrm{~Hz}$ bandstop filter are implemented digitally to suppress motor and grid electromagnetic noise. The RTI samples the code at 1 milliseconds, while the output voltage is logged in intervals of 5 seconds.

\section{MODEL IDENTIFICATION METHODOLOGY}

Given that the temperature measurement data, $\mathrm{T}$, is a time varying parameter available at specific fixed locations, the underlying physical law governing the heat distribution can be presented in form of a PDE. Here we explain the steps taken to identify this PDE.

After reordering the experimental data from $m$ locations at $n$ time samples as a column vector, $\mathrm{T}_{m n \times 1}$, both time and spatial derivatives of the measurements are numerically calculated. The governing dynamic PDE can then be presented as:

$$
T_{t}=f\left(z, T, T^{2}, \ldots, T_{z}, T T_{z}, T_{z z}, \ldots\right)
$$

where $\mathrm{T}_{t}$ denotes the time derivative and $\mathrm{T}_{z}, \mathrm{~T}_{z z}$ etc. denote the derivatives of the temperature to the spatial coordinate $\mathrm{z}$. Subsequently, an over-determined matrix of linear and nonlinear functions of the state $\mathrm{T}_{m n \times 1}$ and the spatial derivatives is constructed, denoted as $\mathrm{S}$ :

$$
S=\left[\begin{array}{ccccccc}
\mid & \mid & \mid & \mid & \mid & \mid & \mid \\
T & T^{2} & \ldots & T_{z} & T T_{z} & T_{z z} & \cdots \\
\mid & \mid & \mid & \mid & \mid & \mid & \mid
\end{array}\right]_{m n \times p}
$$

in which each column represents a possible term for the model. Although the architecture of this set is arbitrary, adopting fundamental functions such as polynomials and trigonometric functions is a common practice. In this regard, expert knowledge and prior information about the system can help choose the appropriate type of nonlinearity, e.g. in terms of relevant orders of polynomials or derivatives.
Once the collection is built, expression (2) is simplified as a linear PDE model [2]:

$$
T_{t}=S \xi
$$

where $\xi$ is a corresponding regressor. Considering that only a few functions in matrix $S$ are likely to appear in the final model, $\xi_{p \times 1}$ is expected to be a sparse vector of coefficients. In other words, most of the parameters in the collection will have a zero weight and will not appear in the model.

As the level of correlation between the columns of matrix $\mathrm{S}$ is high, many regression algorithms such as Lasso have difficulties detecting the correct coefficients when finding the solution to this over-determined linear system of equations [5]. Previous studies have proposed to deal with this problem by replacing Lasso with ridge regression [5], or by reformulating the optimization problem as group Lasso [7]. Group Lasso [9] handles the problem by minimizing the number of active groups of variables, where the variables inside each group are either all zero or all non-zero, with no sparsity considered in groups with non-zero parameters. In contrast, a sparse group Lasso considers groups sparsity and individual feature sparsity [14] and [15]. Correspondingly, we define our optimization problem using the sparse group Lasso. Having the $\mathrm{S}$ matrix broken down to $\mathrm{G}$ submatrices, $\xi^{*}$ is chosen such that it minimizes:

$$
\begin{array}{r}
\xi^{*}=\underset{\xi}{\arg \min }\left(\left\|T_{t}-\sum_{g=1}^{G} S_{g} \xi_{g}\right\|_{2}^{2}\right. \\
+\lambda_{1}\|\xi\|_{1} \\
\left.+\lambda_{2} \sum_{g=1}^{G}\left\|\xi_{g}\right\|_{2}\right)
\end{array}
$$

where $\mathrm{S}_{g}$ is the submatrix of $\mathrm{S}$ with columns corresponding to the terms in group $g$ and $\xi_{g}$ is the coefficient vector of that group. The coefficients $\lambda_{1}$ and $\lambda_{2}$ are the individual and group sparsity regularization parameters, respectively. The higher the values of $\lambda_{1}$ and $\lambda_{2}$, the higher will be the number of zero components in the solution matrix. Correspondingly, the optimal value of these coefficients is found by balancing the number of non-zero components in the solution matrix against the accuracy of the final solution. The length of the groups is chosen based on the assumption on the number of nonzero terms of the solution $\xi^{*}$. For instance if having two nonzero terms is desired, the number of the groups would be $\frac{p}{2}$, each consisting of two terms. Note that both matrices $\mathrm{S}_{g}$ and $\mathrm{T}_{t}$ are normalized to have unit length, so that scaling differences between groups do not affect the results of the algorithm. Once the vector of the arguments of minima $\xi^{*}$ becomes available, its nonzero components are summed to structure the PDE that defines the underlying physical law. 


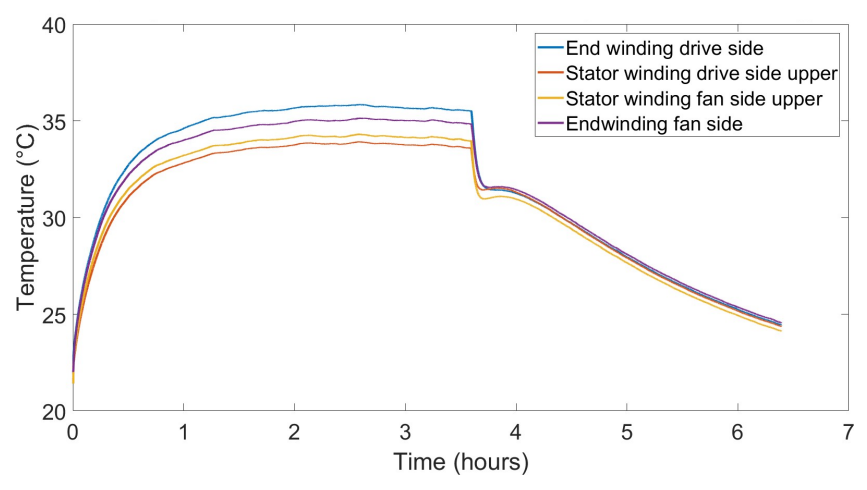

Fig. 3. Temperature profiles with active cooling of the motor windings. For sensor locations, see Fig. 2.

\section{RESULTS AND DisCUSSION}

\section{A. Identification data}

Figures 3 and 4 illustrate the two sets of temperature data collected from the stator winding based on the experiments explained in Section II. Figure 5 shows a zoom of the acquired data in Fig. 4. By comparing Fig. 3 with Fig. 5, it can be observed that the temperature difference between the four sensors in the two sets is not significant. These two data sets are used to assess the efficiency of the identification methodology. We use the data prior to the shut off of the motor: i.e. the data of approximately 3.5 hours of heating for the forced cooling data set of Fig. 3 and 6.5 hours of heating in Fig.4. The reason for this is that we want to identify the internal heat source.

The rms phase current, $\mathrm{I}_{\text {phase,rms }}$, was measured during the operation of the induction machine as being $4.8 \mathrm{~A}$, from which the Joule loss in one phase, $\mathrm{P}_{\mathrm{Cu}, 1 \text { phase }}$, can be deduced:

$$
P_{\mathrm{Cu}, 1 \mathrm{phase}}=R_{s} \times I_{\text {phase,rms }}^{2}=14.4 \mathrm{~W}
$$

where $R_{s}$ is the stator resistance given in Table I. Therefore, the Joule losses within one slot, $\mathrm{P}_{\mathrm{Cu}, 1 \text { slot }}$, can be calculated as:

$$
P_{\mathrm{Cu}, 1 \text { slot }}=\frac{P_{\mathrm{Cu}, 1 \text { phase }}}{N_{\text {slots }}}=1.2 \mathrm{~W}
$$

where $\mathrm{N}_{\text {slots }}=12$ is the total number of slots per phase. As the volume of one stator slot, $\mathrm{V}_{\text {slot }}$, has been estimated to be equal to $2.576 \cdot 10^{-5} \mathrm{~m}^{3}$, the heat source per unit volume, $\mathrm{q}$, can be expressed as follows:

$$
q=\frac{P_{\mathrm{Cu}, 1 \text { slot }}}{V_{\text {slot }}}=4.658 \cdot 10^{4} \frac{\mathrm{W}}{\mathrm{m}^{3}}
$$

This heat source value is used as part of the analysis in the next section to evaluate the accuracy of the model identification results.

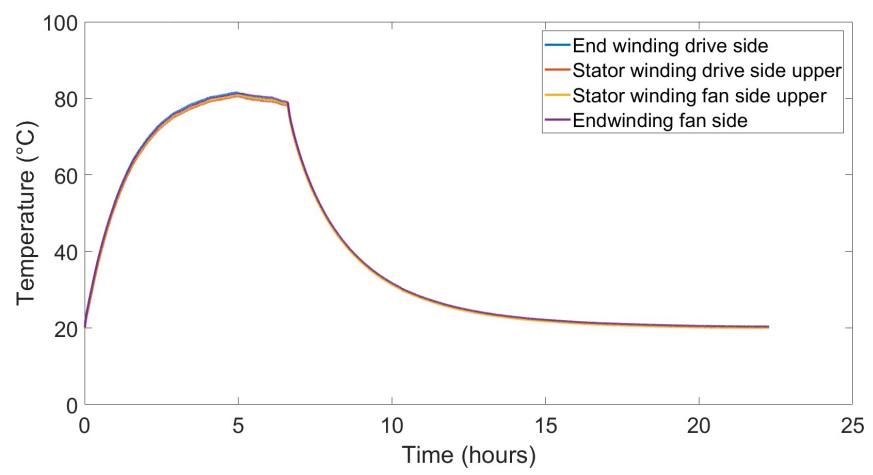

Fig. 4. Temperature profiles with no active cooling of the motor windings. For sensor locations, see Fig. 2.

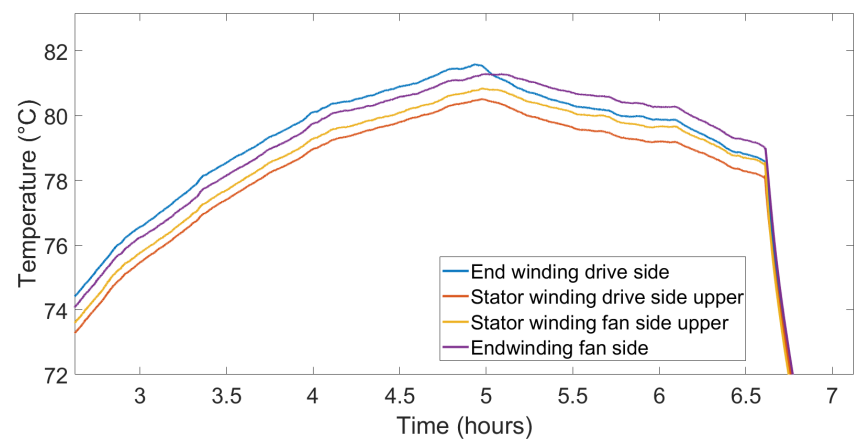

Fig. 5. Magnified temperature profiles with no active cooling of the motor windings. For sensor locations, see Fig. 2.

\section{B. Identification results}

1) Identified coefficients: The search space is represented by considering temperature vector $\mathrm{T}_{m n \times 1}$ with $\mathrm{m}=4$ (sensor locations) and $\mathrm{n}=4500$ samples in case of no active cooling and 2500 samples in case of active motor cooling, up to the second power, its derivatives up to the third order, as well as the different possible combination of their multiplications. A pseudo second order accurate scheme is used to handle unequally spaced data obtained from the four sensors, for for the numerical differentiation (e.g. calculation of $\mathrm{T}_{z}$ ) when building the matrix $\mathrm{S}$ from (3). In order to solve (5), the selected total number of groups (G) equals to 7 and the values of $\lambda_{1}$ and $\lambda_{2}$ are $10^{0}$ and $10^{-2}$ respectively.

The identification yields two nonzero coefficients in the model, namely one coefficient $\left(c_{1}\right)$ relating to the second order derivative of $\mathrm{T}$ in the axial direction, and one constant term $\left(c_{2}\right)$ :

$$
T_{t}=c_{1} T_{z z}+c_{2}
$$

The coefficients obtained using the first data set (Fig. 3) and second data set (Fig. 4), are presented in Table II showing acceptable agreement to each other. 
TABLE II

IDENTIFIED COEFFICIENTS

\begin{tabular}{|c|c|c|}
\hline & $\mathrm{c}_{1}(\mathrm{~J} / \mathrm{kg} . \mathrm{K})$ & $\mathrm{c}_{2}(\mathrm{~J} / \mathrm{kg} . \mathrm{K})$ \\
\hline First data set & $8.163 \cdot 10^{-5}$ & $1.59 \cdot 10^{-2}$ \\
Second data set & $7.867 \cdot 10^{-5}$ & $1.63 \cdot 10^{-2}$ \\
\hline
\end{tabular}

TABLE III

WINDING MATERIAL PROPERTIES

\begin{tabular}{|c|c|c|}
\hline & Copper & Resin \\
\hline Thermal conductivity $k(\mathrm{~J} / \mathrm{m} . \mathrm{K})$ & 385.2 & 0.85 \\
Specific heat capacity $c_{p}(\mathrm{~J} / \mathrm{kg} . \mathrm{K})$ & 386 & 1700 \\
Mass density $\rho\left(\mathrm{kg} / \mathrm{m}^{3}\right)$ & 8890 & 1766 \\
\hline Packing factor & \multicolumn{2}{|c|}{0.85} \\
\hline
\end{tabular}

2) Comparison with theory: The heat equation is given by the following PDE [16]:

$$
\rho c_{p} T_{t}=\nabla(k \nabla T)+q
$$

Therefore, considering one dimensional heat transfer in the axial direction, expression (10) can be rewritten as follows:

$$
T_{t}=\frac{k}{\rho c_{p}} T_{z z}+\frac{1}{\rho c_{p}} q
$$

As mentioned previously, the windings are embedded in a resin matrix inside the stator slot. Therefore, the weighted average of thermal properties of these elements is used to check the validity of the coefficients. For example, the weighted thermal conductivity is calculated using (12), where PF denotes the packing factor. The estimated packing factor as well as the properties, obtained from the literature, are presented in Table III [17]. The same transformation with packing factor can be applied on the other properties $\rho$ and $\mathrm{c}_{p}$.

$$
k=P F \cdot k_{C u}+(1-P F) \cdot k_{R e s i n}
$$

By substituting the known values of $k, \rho, c_{p}$ and $q$, expression (11) yields to the coefficients in Table IV.

The heat equation formulated in (11) confirms the validity of expression (9), meaning that the sparsity regression algorithm has determined the physical law of heat distribution accurately. Furthermore, coefficient values in Table IV agree with those in Table II.

Comparing expressions (11) and (9), it can be noted that the sparse-regression technique has estimated the thermal diffusivity parameter in the axial direction, $\alpha_{z}=k / \rho c_{p}$, through coefficient $c_{1}$ (with values in Table II). This is particularly

TABLE IV

THEORETICAL COEFFICIENTS

\begin{tabular}{|c|c|}
\hline $\mathrm{c}_{1}(\mathrm{~J} / \mathrm{kg} . \mathrm{K})$ & $\mathrm{c}_{2}(\mathrm{~J} / \mathrm{kg} . \mathrm{K})$ \\
\hline $7.182 \cdot 10^{-5}$ & $1.02 \cdot 10^{-2}$ \\
\hline
\end{tabular}

very beneficial because this parameter can be used to estimate $k$ in cases where $\mathrm{c}_{p}$ and $\rho$ are known.

One remarkable observation to emerge from the identification comparison is the correlation between the PDEs obtained from both forced cooled and not forced cooled data sets, suggesting the ability of the sparse regression method to recover the PDE of a system for varying boundary conditions.

\section{CONClusion}

In this work we investigated the possibility of identifying physical laws in terms of PDEs using a sparse regression technique based solely on input-output data analysis. Accordingly, we obtained the heat equation using a modified version of the sparse regression scheme. The experimental data consisted of temperature measurements collected from experiments conducted with a TEFC induction machine, both with and without active cooling. The results were consistent using both the data sets, confirming the reliability of this method when applied on experimental data. In addition, the possibility to recover the PDE of the system for varying boundary conditions was observed. Moreover, it was shown that important thermal properties such as thermal conductivity can be estimated through the identified heat equation coefficients. For future research, experimental investigations with additional temperature measurements along the radial direction within the induction machine can reveal the efficacy of the method applied to more complex PDEs. Furthermore the application of sparsity-based approaches on other experimental data sets are recommended.

\section{REFERENCES}

[1] M. Schmidt and H. Lipson, "Distilling free-form natural laws from experimental data," science, vol. 324, no. 5923, pp. 81-85, 2009.

[2] S. L. Brunton, J. L. Proctor, and J. N. Kutz, "Discovering governing equations from data by sparse identification of nonlinear dynamical systems," Proceedings of the National Academy of Sciences, vol. 113, no. 15, pp. 3932-3937, 2016.

[3] M. Quade, M. Abel, J. N. Kutz, and S. L. Brunton, "Sparse identification of nonlinear dynamics for rapid model recovery," arXiv preprint arXiv:1803.00894, 2018

[4] E. Kaiser, J. N. Kutz, and S. L. Brunton, "Sparse identification of nonlinear dynamics for model predictive control in the low-data limit," arXiv preprint arXiv:1711.05501, 2017.

[5] S. H. Rudy, S. L. Brunton, J. L. Proctor, and J. N. Kutz, "Data-driven discovery of partial differential equations," Science Advances, vol. 3, no. 4, p. e1602614, 2017

[6] H. Schaeffer, "Learning partial differential equations via data discovery and sparse optimization," Proc. R. Soc. A, vol. 473, no. 2197, p. 20160446, 2017.

[7] S. Rudy, A. Alla, S. L. Brunton, and J. N. Kutz, "Data-driven identification of parametric partial differential equations," arXiv preprint arXiv:1806.00732, 2018.

[8] R. Tibshirani, "Regression shrinkage and selection via the lasso," Journal of the Royal Statistical Society. Series B (Methodological), pp. 267-288, 1996.

[9] M. Yuan and Y. Lin, "Model selection and estimation in regression with grouped variables," Journal of the Royal Statistical Society: Series B (Statistical Methodology), vol. 68, no. 1, pp. 49-67, 2006.

[10] H. W. Engl and J. Zou, "A new approach to convergence rate analysis of tikhonov regularization for parameter identification in heat conduction," Inverse Problems, vol. 16, no. 6, p. 1907, 2000.

[11] V. Solo, "Identification of a noisy stochastic heat equation with the em algorithm," in Decision and Control, 2002, Proceedings of the 41st IEEE Conference on, vol. 4. IEEE, 2002, pp. 4505-4508. 
[12] I. E. Commission et al., "Iec 60751: 2008," Industrial platinum resistance thermometers and platinum temperature sensors, 2008.

[13] H. Chandra, S. W. Allen, S. W. Oberloier, N. Bihari, J. Gwamuri, and J. M. Pearce, "Open-source automated mapping four-point probe," Materials, vol. 10, no. 2, p. 110, 2017.

[14] N. Simon, J. Friedman, T. Hastie, and R. Tibshirani, "A sparse-group lasso," Journal of Computational and Graphical Statistics, vol. 22, no. 2, pp. 231-245, 2013.

[15] J. Friedman, T. Hastie, and R. Tibshirani, "A note on the group lasso and a sparse group lasso," arXiv preprint arXiv:1001.0736, 2010.

[16] J. R. Welty, C. E. Wicks, G. Rorrer, and R. E. Wilson, Fundamentals of momentum, heat, and mass transfer. John Wiley \& Sons, 2009.

[17] N. Simpson, R. Wrobel, and P. H. Mellor, "Estimation of equivalent thermal parameters of impregnated electrical windings," IEEE Transactions on Industry Applications, vol. 49, no. 6, pp. 2505-2515, 2013. 